\title{
Sexual behavior of HIV-positive adults not accessing HIV treatment in Mombasa, Kenya: Defining their prevention needs
}

\author{
Avina Sarna ${ }^{1 *}$, Stanley Luchters ${ }^{2}$, Melissa Pickett ${ }^{3}$, Matthew Chersich $^{2,4}$, Jerry Okal ${ }^{5}$, Scott Geibel ${ }^{5}$, Nzioki Kingola ${ }^{6}$ \\ and Marleen Temmerman ${ }^{2}$
}

\begin{abstract}
Background: HIV spread continues at high rates from infected persons to their sexual partners. In 2009, an estimated 2.6 million new infections occurred globally. People living with HIV (PLHIV) receiving treatment are in contact with health workers and therefore exposed to prevention messages. By contrast, PLHIV not receiving ART often fall outside the ambit of prevention programs. There is little information on their sexual risk behaviors. This study in Mombasa Kenya therefore explored sexual behaviors of PLHIV not receiving any HIV treatment.
\end{abstract}

Results: Using modified targeted snowball sampling, 698 PLHIV were recruited through community health workers and HIV-positive peer counsellors. Of the 59.2\% sexually-active PLHIV, 24.5\% reported multiple sexual partners. Of all sexual partners, $10.2 \%$ were HIV negative, while $74.5 \%$ were of unknown HIV status. Overall, unprotected sex occurred in 52\% of sexual partnerships; notably with $32 \%$ of HIV-negative partners and $54 \%$ of partners of unknown HIV status in the last 6 months. Multivariate analysis, controlling for intra-client clustering, showed nondisclosure of HIV status (AOR: 2.38, 95\% Cl: 1.47-3.84, $\mathrm{p}<0.001$ ); experiencing moderate levels of perceived stigma (AOR: 2.94, 95\%Cl: 1.50-5.75, $\mathrm{p}=0.002$ ); and believing condoms reduce sexual pleasure (AOR: $2.81,95 \% \mathrm{Cl}: 1.60-4.91$, $p<0.001)$ were independently associated with unsafe sex. Unsafe sex was also higher in those using contraceptive methods other than condoms (AOR: 5.47, 95\%Cl: 2.57-11.65, $\mathrm{p}<0.001$ ); or no method (AOR: 3.99, 95\%Cl: 2.06-7.75, $\mathrm{p}<0.001$ ), compared to condom users.

Conclusions: High-risk sexual behaviors are common among PLHIV not accessing treatment services, raising the risk of HIV transmission to discordant partners. This population can be identified and reached in the community. Prevention programs need to urgently bring this population into the ambit of prevention and care services. Moreover, beginning HIV treatment earlier might assist in bringing this group into contact with providers and HIV prevention services, and in reducing risk behaviors.

Keywords: PLHIV, Prevention of sexual transmission of HIV, Sexual behavior, Unsafe sex, Africa

\section{Background}

HIV transmission remains a significant global concern; in 2009 there were an estimated 2.6 million new infections globally [1]. At the end of 2009 , about $36 \%$ of the 15 million people in need of antiretroviral treatment (ART) in low- and middle income countries were receiving ART [1].

\footnotetext{
* Correspondence: asarna@popcouncil.org

'Population Council, 142 Golf Links, New Delhi 110048, India

Full list of author information is available at the end of the article
}

People living with HIV (PLHIV) who receive ART are in regular contact with health workers and presumably exposed to prevention messages and commodities. Indeed, several studies have documented a reduction in sexual risk behaviors among PLHIV after initiating ART [2-6]. At the same time, studies have shown that PLHIV accessing HIV care services, but not receiving ART, have higher sexual risk behaviors and unprotected sex than those taking ART, even though both groups have contact with health workers and exposure to prevention messages [7-10]. A major gap, however, is evidence

\section{Biomed Central}


about the patterns of sexual behavior among PLHIV in the community who are not receiving ART and are either accessing HIV care services infrequently or not at all. Although newly diagnosed HIV-positive persons are advised to visit treatment centres for routine follow-up, many PLHIV choose not to. HIV related stigma, denial and disclosure concerns constitute important barriers to accessing care $[11,12]$. The only contact with health services for these people might well be post-test counselling at the time of testing HIV-positive. At the same time, PLHIV are also exposed to HIV prevention messages through mass media and community awareness programs that presumably also influence their knowledge and behaviors.

Studies of the determinants of unprotected sex in HIV-infected people suggest that a range of factors can operate individually or interact to influence sexual behavior [13]. Intention and self-efficacy regarding safe sex; $[14,15]$ myths around condom use; dilemmas around disclosure of HIV status to partner(s) and fears of subsequent rejection; [14,16-20] and motivation to protect partners as well as themselves against re-infection with a new HIV strain or another sexually transmitted infection play an important role in effecting safe sex $[13,20]$. Partner attitudes and willingness to use condoms, complicated by partner status and willingness to be tested for HIV add further dimensions to safe sex practices $[4,18,19,21]$. Furthermore, a desire for children may lead to PLHIV ignoring the risks of unprotected sex $[19,22,23]$.

In Kenya, in 2009, an estimated 1.3 to 1.6 million persons were living with HIV and an estimated $40 \%$ of PLHIV with advanced disease who are eligible for treatment were not receiving ART $[1,24]$. At the same time a large number of PLHIV do not yet require ART. Many of these PLHIV are likely to be outside the ambit of regular health care and prevention services. An estimated 100,000 new HIV infections occurred in 2009 in Kenya, highlighting the need for prevention efforts to focus on sexual risk behaviors of PLHIV, including those not accessing HIV care services. In this paper we examine the sexual risk behaviors of PLHIV in the community who were not receiving ART or co-trimoxazole prophylaxis.

\section{Methods}

Study participants were recruited for a cross-sectional survey, using modified targeted snowball sampling that uses outreach workers to recruit participants from identified geographic areas and populations of interest $[25,26]$. In classical snowball sampling a small number of individuals (typically between 4-6 persons initially) from a particular group of interest are identified, who then serve as 'seeds' to identify and recruit peers, that is, individuals who engage in the same type of risk behaviors, to be included into the study sample [27]. These, initial 'seeds' are often selected by program or study staff via convenience sampling. 'Seeds' can recruit an unlimited number of peers from their network till the desired sample size is achieved or sample saturation takes place. A drawback of this method is that the sample obtained is influenced by characteristics of the initial seeds, the size of their personal network and their ability to reach more cooperative subjects, with a possibility of sampling bias [27]. By contrast, modified targeted sampling, aims to overcome some of the limitations of snowball sampling by including an initial ethnographic assessment aimed at identifying the various networks or subgroups that might exist in a given setting [25]. Participants are then recruited through the active efforts of street outreach workers, using snow-ball sampling. CHWs and PTC counsellors are familiar with the community they serve, the socio-demographic profile of the community and clients, and can help reach PLHIV; we used this cadre of health workers to recruit our study sample. Health workers identified PLHIV in their community and asked these PLHIV to bring in others they knew. As our previous study showed us that PLHIV in Mombasa are relatively isolated due to stigma and disclosure concerns [28], health workers were permitted to add new 'seeds' if PLHIV were unable to bring in peers.

Participants were recruited by community health workers (CHWs) and HIV-positive peer counselors (PCs) from post-test clubs. To reduce biases related to the recruiter and the initial sample, especially over representation of more cooperative subjects and respondents with larger networks, the number of clients each health worker could bring into the study was restricted. Four CHWs from each of Mombasa's four districts ( $\mathrm{n}=$ 16) were each asked to recruit 20 PLHIV; and five PCs from each of eight post-test centers $(n=40)$ across the four districts were each tasked with recruiting 12 PLHIV. HIV-positive adults who were 18 years or older, not currently taking ART or co-trimoxazole prophylaxis were eligible to participate.

Recruitment followed a detailed protocol on approaching PLHIV, maintaining confidentiality and verifying the participant's HIV-positive status by checking the referral card issued by a VCT center, or HIV clinic registration card or HIV/CD4 cell test results. Each participant received Ksh 200 (1USD $=+/-75 \mathrm{Ksh}$ ) as compensation for their time and transport. CHWs and PCs received Ksh 100 per participant recruited to cover their transport costs. Ethical approval was obtained from the Kenyatta National Hospital's Ethics Committee and Institutional Review Board of the Population Council. Written informed consent was obtained from all participants. After data collection was completed, project staff 
worked with CHWs and PTC counsellors to counsel each of the clients they had recruited to return to the HIV clinic for further follow up, care and ART.

Data were collected using structured questionnaires administered in Swahili by trained research assistants. Demographic variables were categorized and time since HIV diagnosis was classified as less than 12 months, 1224 months and $>24$ months. Contraception was categorized as: male/female condoms for contraception, other family planning (FP) methods (intra-uterine device, hormonal methods, permanent methods, diaphragm, foam/jelly, or rhythm) and no contraception. Disclosure of HIV status to a sexual partner was recorded as a binary yes/no variable. Perceived stigma was assessed using an adapted Berger's Stigma Scale (Cronbach's alpha of adapted scale: 0.81 ) and was categorized as minimal or low (16-40), moderate (41-52) or high stigma (53-64) [29,30]. The recall reference period for sexual behavior was the previous 6 months, with data collected on: having had sexual intercourse, number of sexual partners, type of partners, partner's HIV status and disclosure of own status to partners. A regular partner was defined as a spouse or cohabiting partner, or a long-term friend with whom the respondent has sex frequently. A partner with whom the respondent was not living and had sex once or rarely was classified as a casual partner. Commercial or transactional partners were those where money or gifts were exchanged for sex.

To assess transmission concerns, participants were asked a binary question: "Are you worried about transmitting HIV to this partner?" Attitudes to condom use were assessed with six statements: "I am tired of always having to make sure that I use a condom every time I have sex","using condom reduces physical pleasure from sex","if a cure were discovered I would stop using condom", "using a condom takes away the romance from sex", "condoms are effective in preventing HIV and STDs" and "condom should only be used to prevent pregnancy and not HIV"- responses were scored on a 4point Likert scale, ranging from strongly agree, agree, disagree, to strongly disagree. Respondents were also given the option of saying don't know. These statements were adapted from other studies $[13,31,32]$ and pretested, validated and used in a previous study with PLHIV in Mombasa [2,19]. STI events were selfreported episodes of genital discharge or genital ulcer in the last 6 months (laboratory confirmation was unavailable). Participants were asked about the number of biological children they had. Fertility intentions were assessed by asking participants about the intention to have children in the future. Unprotected sex (UPS) was defined as inconsistent condom use with any partner in the past 6 months. Unsafe sex (US), a subset of UPS and the primary outcome for multivariate analysis to determine predictors, was defined as inconsistent condom use with HIV-negative or unknown status partners in the last 6 months. UPS at last sex and US at last sex were also reported.

\section{Data management and statistical analysis}

Data were entered into handheld computers (Dell Axim $\times 51)$ and then uploaded into Microsoft Access 2003 using Perseus 7.0.044 software. The data were analyzed on two levels (respondent-level and partner-level) using Intercooled Stata 8.0 (Stata Corporation, College Station, Texas, USA).

Respondent-level analysis compared demographic and behavioral characteristics of male and female participants. Unpaired Student's $t$ test and the MannWhitney $U$ test compared continuous variables with normal or non-normal distributions respectively, and a chi-square test identified differences between categorical variables. Unadjusted Mantel Hanzel odds ratios were reported.

Analysis at the level of sexual partner included data for up to 6 partners for each respondent in the last 6 months. Univariate logistic regression analyses were performed to identify associations between variables of interest and US at 6 months and last sex. Variables significant, at alpha level of 0.05 , on univariate regression were included in the multivariate model [33]. Although sex of the respondent was not associated with unsafe sex in univariate analysis, it was forced into the model as socio-demographic characteristics varied markedly between women and men (Table 1). Also, a priori, disclosure of HIV status and type of partner were included in initial models, based on previous evidence of association with unprotected sex [7,34-37]. As a participant's sexual behavior with one partner may not be independent from her or his behavior with other partners, we controlled for multiple observations on sexual partners reported by the same study participant (intra-client clustering). Multiple partners of the same participant were also included as separate units of analysis. We adjusted the standard errors for clustering on the participant's ID in both univariate and multivariate logistic regression analyses. A main effects model was used to fit the multivariate model [38]. Separate multivariate models were developed for US at 6 months and at last sex.

\section{Results}

Between May and August 2007, 748 PLHIV were identified by CHWs and PCs; 28 persons were found ineligible (receiving treatment) and 720 PLHIV were interviewed. Data from 22 participants were lost due to technical failures with the hand-held computers, leaving data on 698 participants for analysis. 
Table 1 Participant characteristics: HIV-positive adults not receiving ART, Mombasa, 2007

\begin{tabular}{|c|c|c|c|c|}
\hline Variable & Total $(n=698)$ & Males $(n=164)$ & Females $(n=534)$ & $\mathrm{P}^{\mathrm{a}}$ \\
\hline Age: median (IQR) & $33.5(28-39)$ & $34.5(29-42)$ & $33(28-38)$ & $0.02^{\mathrm{b}}$ \\
\hline \multicolumn{5}{|l|}{ Highest education level: $\%(n)$} \\
\hline No education & $7.3(51)$ & $3.7(6)$ & $8.4(45)$ & 0.04 \\
\hline Primary & $59.2(413)$ & $54.9(90)$ & $60.5(323)$ & \\
\hline Secondary & $31.1(217)$ & $38.4(63)$ & $28.8(154)$ & \\
\hline University & $2.4(17)$ & $3.1(5)$ & $2.3(12)$ & \\
\hline \multicolumn{5}{|l|}{ Marital status: $\%(n)$} \\
\hline Married or cohabiting & $34.4(240)$ & $40.9(67)$ & $32.4(173)$ & $<0.001$ \\
\hline Never married & $21.1(147)$ & $32.9(54)$ & $17.4(93)$ & \\
\hline Divorced or separated & $20.4(143)$ & $15.8(26)$ & $21.9(117)$ & \\
\hline Widowed & $24.1(168)$ & $10.3(17)$ & $28.2(151)$ & \\
\hline \multicolumn{5}{|l|}{ Employment status:\% (n) } \\
\hline Employed & $75.9(530)$ & $82.9(136)$ & $73.8(394)$ & 0.02 \\
\hline \multicolumn{5}{|l|}{ Type of HIV testing facility used:\% (n) } \\
\hline Government health facility & $80.7(563)$ & $77.4(127)$ & $81.7(436)$ & $<0.001$ \\
\hline Private medical centre & $15.3(107)$ & $12.2(20)$ & $16.3(87)$ & \\
\hline Other & $4.0(28)$ & $10.4(17)$ & $2.1(11)$ & \\
\hline \multicolumn{5}{|l|}{ Time since diagnosis: $\%(n)^{c}$} \\
\hline 0-11 months & $43.1(301)$ & $50.0(82)$ & $41.0(219)$ & $<0.001$ \\
\hline $12-23$ months & $19.5(136)$ & $22.6(37)$ & $18.5(99)$ & \\
\hline $24+$ months & $33.4(233)$ & $23.2(38)$ & $36.5(195)$ & \\
\hline \multicolumn{5}{|l|}{ Attends HIV clinic: $\%(n)$} \\
\hline Yes & $23.4(163)$ & $16.5(27)$ & $25.5(136)$ & 0.02 \\
\hline No & $76.7(535)$ & $83.5(137)$ & 74.5 (398) & \\
\hline \multicolumn{5}{|l|}{ Perceived level of stigma:\% ( $n)$} \\
\hline Low & $16.2(113)$ & $18.9(31)$ & $15.4(82)$ & 0.5 \\
\hline Moderate & $68.8(480)$ & $67.7(111)$ & $69.1(369)$ & \\
\hline High & $15.0(105)$ & $13.4(22)$ & $15.5(83)$ & \\
\hline \multicolumn{5}{|l|}{ Drink alcohol weekly: $\%(n)$} \\
\hline Yes & $26.9(188)$ & $34.2(56)$ & $24.7(132)$ & 0.02 \\
\hline \multicolumn{5}{|l|}{ Has ever used drugs: $\%(n)$} \\
\hline Yes & $31.5(220)$ & $68.5(104)$ & $21.7(116)$ & $<0.001$ \\
\hline \multicolumn{5}{|l|}{ Have biological children: $\%(n)$} \\
\hline Yes & $81.7(570)$ & $65.9(108)$ & $86.5(462)$ & $<0.001$ \\
\hline \multicolumn{5}{|l|}{ Want to have children: $\%(n)$} \\
\hline No & $74.8(522)$ & $61.6(101)$ & $78.8(421)$ & $<0.001$ \\
\hline \multicolumn{5}{|l|}{ Using family planning method: $\%(n)^{d}$} \\
\hline No & $54.8(280)$ & $45.5(49)$ & $56.2(237)$ & 0.072 \\
\hline \multicolumn{5}{|l|}{ Reported correct knowledge: $\%(n)$} \\
\hline HIV cannot spread through mosquitoes & $72.8(508)$ & $72.6(119)$ & $72.8(389)$ & 0.93 \\
\hline HIV cannot spread through shared utensils & $84.7(591)$ & $83.5(137)$ & $85.0(454)$ & 0.62 \\
\hline HIV can be transmitted from a mother to child & $91.5(639)$ & $90.2(148)$ & $91.9(491)$ & 0.70 \\
\hline Treatment can reduce mother-to-child transmission & $61.1(425)$ & $55.5(91)$ & $62.8(334)$ & 0.20 \\
\hline HIV+ person can be re-infected with a new virus & $68.6(479)$ & $64.6(106)$ & 69.9 (373) & 0.45 \\
\hline
\end{tabular}

${ }^{\mathrm{a}} \mathrm{X}^{2}$ test unless indicated

${ }^{\mathrm{b}}$ Mann-Whitney $U$ test

${ }^{c} n=671 ; 28$ respondents did not provide information on the time since diagnosis

d of those not wanting children/more children

ART: antiretroviral therapy; IQR: interquartile range 
Median age of participants was 33.5 years $(\mathrm{IQR}=28$ $33)$. Twenty-three percent $(163 / 698)$ of participants reported visiting HIV clinics (34.4\% visited monthly, $16.6 \%$ every two to six months, $20.2 \%$ when sick and $28.8 \%$ off and on). Differences were observed in sociodemographic characteristics between female and male respondents [Table 1]. Women were more likely than men to be widowed (OR 3.40; 95\%CI: 1.98-5.88; p < 0.001); to attend HIV clinic (OR 1.73; 95\%CI: 1.10-2.74; $\mathrm{p}=0.017)$ and be unemployed (OR 1.73; 95\%CI: 1.10 $2.71 ; \mathrm{p}=0.018)$. Women were also less likely to drink alcohol each week (OR: 0.63; 95\%CI: 0.43-0.93; p = 0.017 ) or to report ever using drugs (OR: 0.16 ; $95 \% \mathrm{CI}$ : 0.11-0.24; $\mathrm{p}<0.001)$. Women knew their HIV-status for longer periods than men. Participants recruited by CHWs and by PCs had a similar age, sex, education and employment status (data not shown).

Participants were asked about their reasons for not taking ART; multiple responses were permitted. About a quarter $(27.9 \% ; \mathrm{n}=195)$ reported high CD4 cell counts that made them ineligible for ART; $16.8 \%(\mathrm{n}=117)$ did not want to start ART; $11.2 \%(\mathrm{n}=78)$ reported they were afraid of side-effects; $7.7 \%(\mathrm{n}=54)$ did not know where to access treatment; $2.9 \%(\mathrm{n}=20)$ complained that treatment was expensive; $2.4 \%(\mathrm{n}=17)$ were taking herbal remedies and $2.1 \%(\mathrm{n}=15)$ had unfavourable beliefs about ART, such as, 'ARVs can make you mad', 'ARVs kill you faster', 'ARV are brought by donors when they stop it will be the end of your life', and ARVs make you sicker'.

\section{Sexual activity}

In the 6 months preceding the survey, 59.2\% percent of participants were sexually active; similar in females and males [Table 2].

Males were more likely than female participants to report multiple partners (OR: 3.67; 95\%CI 2.18-6.18; p < 0.001 ) in the last 6 months. Sexually-active male respondents $(90 / 164)$ reported a total of 179 sexual partners and female respondents (320/534) reported a total of 437 sexual partners over the reference period [Table 2].

While the majority of male (84.4\%) and female participants $(98.8 \%)$ reported heterosexual partners, $15.5 \%$ of males $(14 / 90)$ and $1.2 \%$ of females $(n=4 / 320)$ reported same sex partners in the last 6 months [Table 2]. Over a quarter of men's sexual partners were males $(26.8 \%$; 48/179).

Twenty percent of male participants reported a mix of sexual partners (regular/casual/transactional) compared to $9.7 \%$ of female participants (OR: 2.33 ; $95 \% \mathrm{CI}: 1.23$ 4.43; $\mathrm{p}<0.01)$. [Table 2] Female participants reported more regular partners than male participants $(72.1 \%$ vs. 50.8\%; OR: 2.50; 95\%CI: 1.73-3.61; $\mathrm{p}<0.001)$ while male participants had more casual (23.5\% vs. $19.7 \%$; OR: 1.25 ;
95\%CI: 0.82-1.90; $\mathrm{p}=0.29)$ and transactional partners $(25.7 \%$ vs. $8.2 \%$; OR: 3.85 ; $95 \%$ CI: $2.35-6.30 ; \mathrm{p}=<$ $0.001)$ than women $(\mathrm{p}<0.001)$ [Table 2].

Three-quarter of all partners were of unknown HIV status, similar for men and women. Female respondents reported higher disclosure rates to partners than male respondents (39.8\% vs. $30.2 \%$; OR: 1.53 ; 95\%CI: 1.09 2.47; $\mathrm{p}=0.02)$ [Table 2].

\section{Prevalence of unprotected sex}

UPS-6 months (inconsistent condom use with any partner in the last 6 months) was reported in over half (51.9\%) the sexual partnerships, more by women than men $(55.2 \%$ vs. $44.1 \%$; OR: 1.56 ; $95 \%$ CI: $1.09-2.21 ; \mathrm{p}=$ 0.01) [Table 3]. Males were more likely to report UPS-6 months with their female partners than their male partners $(52 \%$ vs. $22.9 \%$; OR: 3.63 ; $95 \%$ CI: $1.66-7.95 ; \mathrm{p}=$ 0.001 ). Both sexes were more likely to have UPS-6 months with regular partners compared to casual or transactional partners $(\mathrm{p}<0.001)$. Inconsistent condom use in the last 6 months (US- 6 months) was reported with $31 \%$ of HIV-negative partners (females $30.4 \%$ vs. males 35.3\%; OR: 0.80; 95\%CI: $0.25-2.63 ; \mathrm{p}=0.72$ ) and with $53.8 \%$ of the partners of unknown HIV status (females $57.3 \%$ vs. males $45.0 \%$; OR: 1.64 ; $95 \% \mathrm{CI}$ : 1.07 2.47; $\mathrm{p}=0.02$ ). Patterns of UPS at last sex were similar to those of UPS-6 months (data not shown).

\section{Predictors of unsafe sex (inconsistent condom use with HIV negative or unknown status partners)}

Risk factors associated with US-6 months were explored (Table 4). In univariate analysis, university level education, more than 12 months since HIV diagnosis, nondisclosure of HIV-status, moderate and high levels of internalized stigma, condom-use fatigue, attending a HIV clinic, knowing that re-infection with a new viral strain is possible, believing that condoms reduce pleasure and using non-condom contraceptive methods were associated with higher risk of US-6 months and were included in the initial model.

In multivariate analysis, after controlling for multiple observations relating to different sexual partners reported by the same study participant, non-disclosure of HIV status to a partner (AOR 2.38, 95\%CI: 1.47-3.84; $\mathrm{p}<0.001$ ), experiencing moderate levels of perceived stigma (AOR 2.94, 95\%CI: 1.50-5.75; $\mathrm{p}=0.002$ ), believing condoms reduce sexual pleasure (AOR 2.81, 95\%CI: 1.60-4.91; $\mathrm{p}<0.001)$ or being unsure about condoms reducing pleasure (AOR 8.33, 95\%CI: 2.38-29.09; $\mathrm{p}=$ $0.001)$, using a non-condom contraceptive method (AOR 5.47, 95\%CI: 2.57-11.65; p < 0.001) or not using any contraception (AOR 3.99, 95\%CI: 2.06-7.75; p < 0.001 ) were independently associated with US-6 months. Sex of the respondent, though not significantly 
Table 2 Sexual behavior among HIV-positive adults not receiving ART in Mombasa, Kenya 2007

\begin{tabular}{|c|c|c|c|c|}
\hline \multicolumn{5}{|c|}{ All Respondents } \\
\hline & Total $(n=698)$ & $\begin{array}{c}\text { Males } \\
(n=164)\end{array}$ & $\begin{array}{l}\text { Females } \\
(n=534)\end{array}$ & $\begin{array}{c}P \\
\text { Value }^{a}\end{array}$ \\
\hline $\begin{array}{l}\text { Lifetime no. of } \\
\text { partners: median (IQR) }\end{array}$ & $5(3,10)$ & $14(6,25)$ & $4(3,8)$ & $\begin{array}{c}< \\
0.001\end{array}$ \\
\hline $\begin{array}{l}\text { Sexually active in } \\
\text { past } 6 \text { months: } \%(n)\end{array}$ & $59.2(413)$ & 55.5 (91) & $60.3(322)$ & 0.27 \\
\hline
\end{tabular}

\section{Sexually Active Respondents}

$\begin{array}{ccc}\text { Total } & \text { Male } & \text { Female } \\ (\mathrm{n}=410)^{\mathrm{c}} & (\mathrm{n}=90) & \mathrm{n}=320)\end{array}$

No. of partners in past 6 months: $\%(n)^{d}$

\begin{tabular}{lllc}
\hline One partner & $75.5(308)$ & $54.4(49)$ & $81.5(259)$ \\
\hline $\begin{array}{l}\text { More than one } \\
\text { partner }\end{array}$ & $24.5(100)$ & $45.6(41)$ & $18.6(59)$ \\
\hline
\end{tabular}

Sex of partner:\% (n)

\begin{tabular}{lccc}
\hline Only male & $79.8(327)$ & $12.2(11)$ & $98.8(316)$ \\
\hline Only female & $18.8(77)$ & $84.4(76)$ & $0.3(1)$ \\
\hline $\begin{array}{l}\text { Both male \& } \\
\text { female }\end{array}$ & $1.5(6)$ & $3.3(3)$ & $0.9(3)$ \\
\hline Type of partner:\% (n) & & & $0.001^{\mathrm{e}}$ \\
\hline
\end{tabular}

\begin{tabular}{lccc}
\hline Only regular & $76.3(313)$ & $62.2(56)$ & $80.3(257)$ \\
\hline Only casual & $8.5(35)$ & $11.1(10)$ & $7.8(25)$ \\
\hline Only sex worker & $3.2(13)$ & $6.7(6)$ & $2.2(7)$ \\
\hline Multiple types & $12.0(49)$ & $20.0(18)$ & $9.7(31)$ \\
\hline
\end{tabular}

Sexually Active Respondents (partner level analysis: $n=616$ )

\begin{tabular}{|c|c|c|c|c|}
\hline & $\begin{array}{l}\text { Total number of partners reported by } \\
410 \text { sexually active participants }\end{array}$ & $\begin{array}{c}\text { Number of partners } 90 \\
\text { sexually active men reported }\end{array}$ & $\begin{array}{c}\text { Number of partners } 320 \\
\text { sexually active women reported }\end{array}$ & $\begin{array}{c}\mathrm{P} \\
\text { Value }^{\mathrm{a}}\end{array}$ \\
\hline $\begin{array}{l}\text { Partners of } \\
\text { respondents }\end{array}$ & $n=616$ & $n=179$ & $n=437$ & \\
\hline \multicolumn{5}{|l|}{ Sex of partner:\% (n) } \\
\hline Male & $78.1(481)$ & $26.8(48)$ & 99.1 (433) & \\
\hline Female & $21.9(135)$ & $73.2(131)$ & $0.9(4)$ & $0.001^{\mathrm{e}}$ \\
\hline \multicolumn{5}{|l|}{ Type of partner:\%(n) } \\
\hline Regular & $65.9(406)$ & $50.8(91)$ & $72.1(315)$ & \\
\hline Casual & $20.8(128)$ & $23.5(42)$ & $19.7(86)$ & \\
\hline Sex worker & $13.3(82)$ & $25.7(46)$ & $8.2(36)$ & $\begin{array}{c}<< \\
0.001\end{array}$ \\
\hline \multicolumn{5}{|c|}{ Partner HIV status:\% (n) } \\
\hline Positive & $15.3(94)$ & $17.3(31)$ & $14.4(63)$ & \\
\hline Negative & $10.2(63)$ & $9.5(17)$ & $10.5(46)$ & \\
\hline Unknown & 74.5 (459) & $73.2(131)$ & 75.1 (328) & 0.64 \\
\hline \multicolumn{5}{|l|}{ Disclosure:\% (n) } \\
\hline Partner knows & $37.0(228)$ & $30.2(54)$ & $39.8(174)$ & \\
\hline $\begin{array}{l}\text { Partner does not } \\
\text { know }\end{array}$ & $63.0(388)$ & $69.8(125)$ & $60.2(263)$ & 0.02 \\
\hline
\end{tabular}

\footnotetext{
${ }^{\mathrm{a}} X^{2}$ test unless indicated

${ }^{b} \mathrm{n}=684 ; 14$ respondents were excluded if they did not know, did not respond, or reported $\geq 800$ partners

${ }^{c} n=410 ; 3$ sexually active respondents did not answer further questions about their sexual partners

${ }^{\mathrm{d}} \mathrm{n}=408 ; 2$ respondents did not respond

e Fisher's exact test

$A R T$, antiretroviral therapy; IQR, interquartile range
} 
Table 3 Prevalence of Unprotected Sex in the past 6 months among sexually-active participants (partner level analysis)

\begin{tabular}{|c|c|c|c|}
\hline & $\begin{array}{l}\text { Total number of partners reported by } \\
410 \text { sexually active respondents } \\
n=616\end{array}$ & $\begin{array}{l}\text { UPS- } 6 \text { months Number of partners by } \\
90 \text { sexually active male respondents } \\
n=179\end{array}$ & $\begin{array}{l}\text { Number of partners reported by } 320 \\
\text { sexually active female respondents } \\
n=437\end{array}$ \\
\hline $\begin{array}{l}\text { Total } \\
\text { Unprotected } \\
\text { Sex: } \%(n)\end{array}$ & $51.9(320 / 616)$ & $44.1(79 / 179)$ & $55.2(241 / 437)$ \\
\hline \multicolumn{4}{|c|}{ By sex of partner:\% (n) } \\
\hline Male & $52.4(252 / 481)$ & $22.9(11 / 48)$ & $55.7(241 / 433)$ \\
\hline \multirow[t]{2}{*}{ Female } & $50.4(68 / 135)$ & $52.0(68 / 131)$ & $0(0 / 4)$ \\
\hline & & $p=0.001$ & $p=0.04^{b}$ \\
\hline \multicolumn{4}{|c|}{ By type of partner:\% (n) } \\
\hline Regular & $61.1(248 / 406)$ & $59.3(54 / 91)$ & $61.6(194 / 315)$ \\
\hline Casual & $34.4(44 / 128)$ & $26.2(11 / 42)$ & $38.4(33 / 86)$ \\
\hline \multirow[t]{2}{*}{ Sex worker } & $34.2(28 / 82)$ & $30.4(14 / 46)$ & $38.9(14 / 36)$ \\
\hline & $p<0.001$ & $p<0.001$ & $p<0.001$ \\
\hline \multicolumn{4}{|c|}{ By partner status: $\%(n)$} \\
\hline Positive & $56.4(53 / 94)$ & $45.2(14 / 31)$ & $61.9(39 / 63)$ \\
\hline Negative & $31.8(20 / 63)$ & $35.3(6 / 17)$ & $30.4(14 / 46)$ \\
\hline \multirow[t]{2}{*}{ Unknown } & $53.8(247 / 459)$ & $45.0(59 / 131)$ & $57.3(188 / 328)$ \\
\hline & $p=0.003$ & $p=0.74$ & $p<0.001$ \\
\hline \multicolumn{4}{|c|}{ By disclosure:\% (n) } \\
\hline $\begin{array}{l}\text { Partner } \\
\text { knows }\end{array}$ & $53.1(121 / 228)$ & $38.9(21 / 54)$ & $57.5(100 / 174)$ \\
\hline $\begin{array}{l}\text { Partner } \\
\text { does not }\end{array}$ & $51.3(199 / 388)$ & $46.4(58 / 125)$ & $53.6(141 / 263)$ \\
\hline know & $p=0.67$ & $p=0.35$ & $p=0.43$ \\
\hline
\end{tabular}

${ }^{a} X^{2}$ test unless indicated

${ }^{b}$ Fisher's exact test

ART, antiretroviral therapy

associated with US-6 months in univariate analysis, was associated with US- 6 months on multivariate analysis: female respondents were two times more likely to report US-6 months (AOR 2.10; 95\%CI: 1.13-3.90; p = 0.018) compared to male respondents. University education and time since HIV diagnosis were not associated with US-6 months. Predictors for US-last sex were similar to those for US-6 months. (Data not shown)

\section{Sexually transmitted infections}

Overall, 44\% of participants reported ever having a STI other than HIV. Males were twice as likely to ever report a STI compared to females $(55.9 \%$ vs. $41.0 \%$; OR: 1.82, 95\%CI: $1.27-2.61 ; \mathrm{p}<0.001)$. Of those who ever had a STI, half $(49.5 \%)$ had a STI in the last 6 months. A higher proportion of female participants reported genital discharge (42.9\% vs. $19.7 \%$, OR: 3.06 ; 95\%CI: $1.68-$ $5.55 ; \mathrm{p}<0.001)$ and ulcers (38.2\% vs. $25.5 \%$; OR: 1.80 ; $95 \% \mathrm{CI}: 1.04-3.11 ; \mathrm{p}=0.046)$ in the last six months than men. Of note, $46.5 \%$ of participants reporting a STI informed their regular partners of their infection, but only $13.9 \%$ of those with multiple partners informed other partners.

\section{Other sexual practices}

Twenty nine percent of sexually active respondents reported sexual intercourse with a partner during menstruation (24/90 males and 94/318 females). Of those, $78 \%$ (18/24 males and 74/94 females) inconsistently or never used condoms for sex during menstrual periods. Eighteen percent of sexually active respondents (23/90 males and 50/318 females) reported ever having anal sex. Of those, $80.8 \%$ ( $14 / 23$ males and $45 / 50$ females) inconsistently or never used condoms during anal sex. (Data not shown)

\section{Discussion}

This study, conducted in Mombasa among PLHIV not accessing HIV treatment, shows the population has high levels of unsafe sex. Almost sixty percent of the participants were sexually-active during the last 6 months. This is significantly higher than that reported in our previous study in Mombasa among PLHIV receiving ART (44\%) and PLHIV receiving co-trimoxazole prophylaxis without ART (47\%) [7], and in other studies among PLHIV accessing care services in Cote d' Ivoire (47\%), Uganda (48\%) and Cameroon (47\%) [8,10,39]. 
Table 4 Factors associated with Unsafe Sex in the past 6 months among sexually-active participants, adjusted for intra-client clustering

\begin{tabular}{|c|c|c|c|c|c|}
\hline \multirow[b]{2}{*}{ Variable } & \multicolumn{4}{|c|}{ US-6 months } & \multirow[b]{2}{*}{$P$ value } \\
\hline & Prevalence \% (n) & Crude Odds $(95 \% \mathrm{Cl})$ & $P$ value & Adjusted Odds $(95 \% \mathrm{Cl})$ & \\
\hline \multicolumn{6}{|l|}{ Sex of respondent } \\
\hline Male $(n=179)$ & $36.3(65)$ & 1.0 & - & - & - \\
\hline Female $(n=437)$ & $46.2(202)$ & $1.51(0.88-2.59)$ & 0.14 & $2.10(1.13-3.90)$ & 0.018 \\
\hline \multicolumn{6}{|l|}{ Age } \\
\hline $18-24$ years $(n=106)$ & $42.5(45)$ & $0.85(0.44-1.65)$ & 0.64 & & \\
\hline $25-34$ years $(n=291)$ & $46.4(135)$ & 1.0 & - & & \\
\hline $35-44$ years $(184)$ & $39.7(73)$ & $0.76(0.46-1.26)$ & 0.29 & & \\
\hline $45+(n=35)$ & $40.0(14)$ & $0.77(0.31-1.94)$ & 0.58 & & \\
\hline \multicolumn{6}{|l|}{ Marital status } \\
\hline Married or cohabiting $(\mathrm{n}=232)$ & $44.8(104)$ & 1.0 & - & & \\
\hline Never married $(n=184)$ & $41.3(76)$ & $0.87(0.49-1.51)$ & 0.61 & & \\
\hline Divorced, separated, or widowed $(n=200)$ & $43.5(87)$ & $0.95(0.59-1.53)$ & 0.83 & & \\
\hline \multicolumn{6}{|l|}{ Highest education level } \\
\hline No education $(n=42)$ & $52.4(22)$ & $1.32(0.53-3.26)$ & 0.55 & $0.53(0.20-1.37)$ & 0.19 \\
\hline Primary $(n=378)$ & $45.5(172)$ & 1.0 & - & - & - \\
\hline Secondary $(n=179)$ & $39.1(70)$ & $0.77(0.47-1.26)$ & 0.30 & $1.21(0.63-2.32)$ & 0.56 \\
\hline University $(n=17)$ & $17.7(3)$ & $0.26(0.07-0.98)$ & 0.05 & $0.82(0.22-3.03)$ & 0.77 \\
\hline \multicolumn{6}{|l|}{ Sex of partner } \\
\hline Male $(n=481)$ & $44.3(213)$ & 1.0 & - & & \\
\hline Female $(n=135)$ & $40.0(54)$ & $0.84(0.49-1.42)$ & 0.52 & & \\
\hline \multicolumn{6}{|l|}{ Type of partner } \\
\hline Regular $(n=406)$ & $48.3(196)$ & $1.80(0.88-3.68)$ & 0.11 & & \\
\hline Casual $(n=128)$ & $33.6(43)$ & $0.98(0.41-2.32)$ & 0.96 & & \\
\hline Sex worker $(n=82)$ & $34.2(28)$ & 1.0 & - & & \\
\hline \multicolumn{6}{|l|}{ Attends HIV clinic } \\
\hline Yes $(n=122)$ & $25.6(36)$ & $0.47(0.29-0.78)$ & 0.003 & $0.60(0.34-1.06)$ & 0.08 \\
\hline No $(n=494)$ & $46.8(231)$ & 1.0 & - & 1.0 & - \\
\hline \multicolumn{6}{|l|}{ Time since diagnosis } \\
\hline$<12$ months $(\mathrm{n}=265)$ & $52.8(140)$ & 1.0 & - & - & - \\
\hline $12-24$ months $(n=131)$ & $37.4(49)$ & $0.53(0.30-0.94)$ & 0.03 & $0.61(0.33-1.31)$ & 0.12 \\
\hline 2 months $(n=197)$ & $35.0(69)$ & $0.48(0.28-0.82)$ & 0.01 & $0.74(0.42-1.30)$ & 0.31 \\
\hline \multicolumn{6}{|l|}{ Knowledge: re-infection with new strain } \\
\hline Yes $(n=426)$ & $36.8(157)$ & 1.0 & & 1.0 & \\
\hline No/Does not know $(n=190)$ & $57.9(110)$ & $2.35(1.43-3.86)$ & 0.001 & $1.27(0.71-2.27)$ & 0.41 \\
\hline \multicolumn{6}{|l|}{ Disclosure to partner } \\
\hline Yes $(n=228)$ & $31.1(71)$ & 1.0 & - & - & - \\
\hline No $(n=388)$ & $50.5(196)$ & $2.26(1.52-3.35)$ & $<0.001$ & $2.38(1.47-3.84)$ & $<0.001$ \\
\hline \multicolumn{6}{|l|}{ Transmission concerns } \\
\hline Yes $(n=394)$ & $43.7(172)$ & 1.0 & - & & \\
\hline No $(n=222)$ & $42.8(95)$ & $0.97(0.63-1.49)$ & 0.87 & & \\
\hline \multicolumn{6}{|l|}{ Had STI in past 6 months } \\
\hline Yes $(n=145)$ & $42.8(62)$ & $0.97(0.58-1.63)$ & 0.91 & & \\
\hline No $(n=471)$ & $43.5(205)$ & 1.0 & - - & & \\
\hline \multicolumn{6}{|l|}{ Perceived internalized stigma } \\
\hline Minimal/Low $(n=98)$ & $17.4(17)$ & 1.0 & - & - & - \\
\hline Moderate $(n=431)$ & $46.9(202)$ & $4.20(2.22-7.95)$ & $<0.001$ & $2.94(1.50-5.75)$ & 0.002 \\
\hline High $(n=87)$ & $55.2(48)$ & $5.86(2.60-13.21)$ & $<0.001$ & $1.93(0.74-5.03)$ & 0.18 \\
\hline \multicolumn{6}{|l|}{ Tired of using condoms } \\
\hline Agree $(n=253)$ & $49.8(126)$ & $1.99(1.26-3.14)$ & 0.003 & $1.35(0.80-2.25)$ & 0.25 \\
\hline
\end{tabular}


Table 4 Factors associated with Unsafe Sex in the past 6 months among sexually-active participants, adjusted for intra-client clustering (Continued)

\begin{tabular}{|c|c|c|c|c|c|}
\hline Disagree $(n=319)$ & $33.2(106)$ & 1.0 & - & - & - \\
\hline Do not $\operatorname{know}(n=44)$ & $79.6(35)$ & $7.81(3.31-18.43)$ & $<0.001$ & $5.01(1.78-14.07)$ & 0.002 \\
\hline \multicolumn{6}{|l|}{ Believe condom reduces pleasure } \\
\hline Agree $(n=369)$ & $51.0(188)$ & $2.87(1.81-4.54)$ & $<0.001$ & $2.81(1.60-4.91)$ & $<0.001$ \\
\hline Disagree $(n=222)$ & $26.6(59)$ & 1.0 & - & - & - \\
\hline Ambivalent $(n=25)$ & $80.0(20)$ & $11.1(4.03-30.28)$ & $<0.001$ & $8.33(2.38-29.09)$ & 0.001 \\
\hline \multicolumn{6}{|l|}{ Family planning } \\
\hline Using condom $(n=124)$ & $16.1(20)$ & 1.0 & - & - & - \\
\hline Using other method $(n=117)$ & $53.0(62)$ & $5.9(2.87-11.96)$ & $<0.001$ & $5.47(2.57-11.66)$ & $<0.001$ \\
\hline No family planning $(\mathrm{n}=375$ & $49.3(185)$ & $5.06(2.72-9.42)$ & $<0.001$ & $3.99(2.06-7.75)$ & $<0.001$ \\
\hline \multicolumn{6}{|l|}{ Drink Alcohol Weekly } \\
\hline Yes $(n=251)$ & $46.6(117)$ & $1.25(0.79-1.97)$ & 0.34 & & \\
\hline No $(n=365)$ & $41.1(150)$ & 1.0 & - & & \\
\hline
\end{tabular}

ART, antiretroviral therapy; $\mathrm{Cl}$, confidence interval; STI, sexually transmitted infection

Further, participants reported unprotected sex with more than half their sexual partners, significantly more with regular partners than non-regular partners. This is much higher than that reported among ART-naïve PLHIV in Uganda and South Africa [4,9,37] as well as among PLHIV on ART and those on co-trimoxazole prophylaxis without ART in Mombasa [2,7]. It is of concern that unprotected sex was reported with a third of HIV-negative partners and half of untested partners (people with unknown HIV status). This presents a serious HIV prevention challenge, particularly as 75 percent of the partners were untested and only 37 percent of the PLHIV had disclosed their HIV status to their partners. In a review article, Kalichman et al. (2000), have also reported high levels of unsafe sex with HIVnegative and unknown status partners [17]. Disclosure of HIV serostatus to partners and perceived stigma emerged as independent determinants of safe sex behaviors. It is important to note the intersection of the two determinants where PLHIV are reluctant to disclose for fear of rejection (perceived stigma) which may or may not happen $[18,19]$. Loubiere et al. (2009) and King et al. (2008) also link disclosure of HIV status with safe sex behavior in studies from Cameroon and Uganda $[21,35]$. Our study also highlights the role that the belief that condoms reduce pleasure and condom-use fatigue play in influencing safe sex. Conley and Collins (2005) found condom non-users to be more likely to believe that condom use interferes with pleasure; more commonly among males. Randolph et al. (2007) report similar results on condom use $[40,41]$. Prevention programs need to develop and implement strategies to change attitudes and beliefs about condoms. Further, more than half of the participants who did not want to have children were not using contraception, indicating high levels of unmet family planning need. Although it has been discussed extensively, effective integration of family planning counseling and services into HIV prevention programs has not been implemented and merits urgent action $[42,43]$.

We documented other risky sexual practices such as unprotected sex during menstruation and unprotected anal sex. Sexual exposure to genital blood during menstruation is believed to facilitate transmission of HIV and other STIs $[44,45]$. We also report same sex behaviors among male participants: almost a quarter of all sexual partners reported by male participants were male. It is possible that a MSM peer could have recruited MSM participants. Mombasa has a fairly large population of male sex workers and unprotected anal sex is frequently reported in this population $[46,47]$. Anal intercourse is reported relatively less frequently by women. Fifteen percent of female participants in our study reported ever anal sex and the vast majority did not use condoms. Kalichman et al. (2009) report a 10\% prevalence of heterosexual anal sex reported by women interviewed from community and clinic settings in South Africa [48]. The relatively low prevalence of anal intercourse among heterosexual individuals may be offset by its greater efficiency for transmitting HIV [49]. Health workers need to specifically discuss these forms of risky sexual behaviors during prevention counselling.

The study provides evidence that prevention programs can reach PLHIV who are not accessing HIV care services through community health workers or peer counsellors. About three-quarter of the participants were not accessing any HIV care and support services that they could benefit from; and more than half of them had been tested positive more than 12 months previously and were therefore, more likely to have forgotten any prevention messaging at the time of post-test counselling. This occurred despite the increased availability of 
HIV care services and ART in recent years. Further research is needed to examine why some PLHIV are not accessing HIV care services.

The study is not without limitations. We recruited participants using non-probability modified targeted snowball sampling. Although our sample is not a randomly recruited representative sample, this technique did allow us to reach PLHIV within the community who are otherwise not accessible. We believe we were able to recruit a sufficiently diverse and representative sample for this study. We did not use Respondent Driven Sampling, a technique used commonly for hidden hard-toreach populations such as MSM and injecting drug users, as this sampling method relies heavily on the recruitment of peers through their social networks, and we felt this to be unsuitable for our population and setting. In Mombasa, the network of positive people is small and poorly organized and, our previous study showed that PLHIV were reluctant to reveal their status and were poorly networked, with high levels of internalized stigma. This has also been reported by other African studies $[11,12,28]$. Our study sample consisted of $76 \%$ female participants. There could be several reasons for this: women tend to stay at home and therefore may be more easily contacted by health workers, women may access care earlier than men and so are more likely to know their HIV status, and in general, women make up more than $60 \%$ of the HIV-positive population in subSaharan Africa [1]. Women constituted $64 \%$ and $66 \%$ of our sample in our two previous studies in Mombasa $[2,7]$.

In our data analysis we did not control for clustering at the recruiter level, which could lead to increased variance in reported behaviors. We did not do so because we did not link data on individual recruiters to participants; we recorded only type of recruiters (CHW or PTC counsellor). However, the fact that we found no significant socio-demographic differences between PLHIV recruited by $\mathrm{CHW}$ and those recruited by PCs, and that each health worker could bring in a limited number of participants into the study and health workers were able to reach different risk groups as there are no geographic areas in Mombasa with a concentration of particular high-risk populations, may have reduced the bias due to clustering at the recruiter level. The study relies on self-reported sexual risk and condom use behaviors which may be subject to social desirability and recall bias. For the partner level analysis, we limited the number of partners each participant could describe to a maximum of six in the reference period; this afforded us the ability to obtain more reliable recall and limit the influence of the outliers in the sample. Reviews of validity and reliability of HIV research have, however, found that sexual behavior data are fairly consistent and selfreported data on sexual acts are reasonably congruent, especially for infrequent acts and short recall periods $[50,51]$. However, recent studies using biomarkers to validate self-reported condom use suggest over reporting of condom use and recommend interpreting selfreported behaviors with caution [52]. Over reporting would further raise the level of risk found in this study. Finally, the study would have benefited if a control group of PLHIV on treatment had been included for a comparison of sexual behaviors.

In conclusion, a significantly large number of PLHIV in the community are not accessing ART or HIV care services in Mombasa and high risk sexual behaviors are widely prevalent in this population. HIV programs need to bring this population into the ambit of prevention and care services.

\section{Acknowledgements}

We acknowledge the contribution of our colleagues from the Population Council: Ms Susan Kaai in Nairobi, in proposal development and training and Dr Waimar Tun, in Washington DC, for her inputs into proposal development and review of the report. We recognize the contribution of our team of research interviewers: Nicodemus Kisengese, Mariam Kassim, Jacqueline Chokwe, Dorothy Mubweka, Caleb Muasya and Nancy Kingola. The study could not have been done without a team of dedicated Community Health Workers and Post Test Club Peers led by Chorongo Salee and Peter Kimani respectively, our thanks to them. Lastly, we thank all study participants for their invaluable contribution. This work was undertaken by the Population Council and was funded by the generous support of the American people through the US Agency for International Development (USAID), Cooperative Agreement Award No: HRN-A-00-97-00012-00. The opinions expressed herein are those of the authors and do not necessarily reflect the views of USAID.

\section{Author details}

${ }^{1}$ Population Council, 142 Golf Links, New Delhi 110048, India. ${ }^{2}$ International Centre for Reproductive Health, Department of Obstetrics and Gynaecology, Ghent University, Ghent, Belgium. ${ }^{3}$ UCLA Center for Health Policy Research, Los Angeles, USA. ${ }^{4}$ Centre for Health Policy, School of Public Health, Faculty of Health Sciences, University of the Witwatersrand, Johannesburg, South Africa. ${ }^{5}$ Population Council, Nairobi, Kenya. ${ }^{6}$ International Centre of Reproductive Health, Mombasa, Kenya.

\section{Authors' contributions}

AS was the PI on the study; she contributed to the design of the study, analysis of data and wrote the manuscript. SL participated in the design of the study and contributed to the manuscript. MP conducted the statistical data analysis. MC contributed substantially in reviewing the manuscript and guided data analysis. $\mathrm{JO}$ and NK conducted field research and assisted with contextual data and result interpretation. SG set up the data collection using hand held computers and helped with data interpretation. MT provided overall guidance for the research and manuscript preparation. All authors read and approved the final manuscript.

\section{Competing interests}

The authors declare that they have no competing interests.

Received: 8 September 2011 Accepted: 19 March 2012 Published: 19 March 2012

\section{References}

1. UNAIDS, Global report: UNAIDS report on the global AIDS epidemic 2010 2010 [http://www.unaids.org/globalreport/Global_report.htm]. 
2. Luchters $S$, et al: Safer sexual behaviors after 12 months of antiretroviral treatment in Mombasa, Kenya: a prospective cohort. AIDS Patient Care STDS 2008, 22(7):587-594.

3. Peltzer K, Ramlagan $\mathrm{S}$ : Safer sexual behaviors after 1 year of antiretroviral treatment in KwaZulu Natal, South Africa: a prospective cohort study. Sex Heal 2010, 7(2):135-141

4. Bunnell R, et al: Changes in sexual behavior and risk of HIV transmission after antiretroviral therapy and prevention interventions in rural Uganda. AIDS 2006, 20(1):85-92.

5. Crepaz N, Hart TA, Marks G: Highly Active Antiretroviral Therapy and Sexual Risk Behavior A Meta-analytic Review. JAMA 2004, 292(2):224-236.

6. Wandera $B$, et al: Sexual behaviors over a 3-year period among individuals with advanced HIV/AIDS receiving antiretroviral therapy in an urban HIV clinic in Kampala, Uganda. J Acquir Immune Defic Syndr 2011.

7. Sarna A, et al: Sexual risk behavior and HAART: a comparative study of HIV-infected persons on HAART and on preventive therapy in Kenya. Int J STD AIDS 2008, 19:85-89.

8. Moatti J-P, et al: Access to antiretroviral treatment and sexual behaviors of HIV-infected patients aware of their serostatus in Côte d'Ivoire. AIDS 2003, 17(supplementary 3):S69-577.

9. Eisele TP, et al: High levels of risk behavior among people living with HIV initiating and waiting to start antiretroviral therapy in Cape Town, South Africa. AIDS Behav 2008, 12(4):570-577.

10. Bateganya $\mathrm{M}$, et al: Antiretroviral therapy and sexual behavior: a comparative study between antiretroviral- naive and -experienced patients at an urban HIV/AIDS care and research center in Kampala, Uganda. AIDS Patient Care and STDs 2005, 19(11):760-768.

11. Roura M, et al: Scaling up stigma? The effects of antiretroviral roll-out on stigma and HIV testing. Early evidence from rural Tanzania. Sex Transm Infect 2009, 85(4):308-312.

12. Mshana $G$, et al: Barriers to accessing antiretroviral therapy in Kisesa, Tanzania: a qualitative study of early rural referrals to the national program. AIDS Patient Care STDS 2006, 20(9):649-657.

13. Crepaz N, Marks G: Towards an understanding of sexual risk behavior in people living with HIV: a review of social, psychological, and medical findings. AIDS 2002, 16:135-149.

14. Shiltz M, Sandfort TGM: HIV-positive people, risk and sexual behavior. Social Sci Med 2000, 50(11):1571-1588.

15. Courtney-Quirk $C$, et al: Factors associated with sexual risk behavior among persons living with HIV: Gender and sexual identity group differences. AIDS Behav 2008, 12(5):685-694.

16. Kiene SM, et al: High rates of unprotected sex occurring among HIVpositive individuals in a daily diary study in South Africa: The role of alcohol use. J Acquir Immune Defic Syndr 2008, 49(2):219-226.

17. Kalichman SC: HIV transmission risk behaviors of men and women living with HIV-AIDS: Prevalence, predictors and emerging clinical interventions. Clin Psychological Sci Pract 2000, 7:32-47.

18. Kerrigan $D$, et al: The search for social validation and the sexual behavior of people living with HIV in Rio de Janeiro, Brazil: understanding the role of treatment optimism in context. Social Sci Med 2006, 62(10):2386-2396.

19. Sarna A, et al: Changes in sexual risk taking with antiretroviral treatment: influence of context and gender norms in Mombasa, Kenya. Cult Heal Sex 2009, 11(8):783-797.

20. King $R$, et al: The virus stops with me: HIV-infected Ugandans' motivations in preventing HIV transmission. Soc Sci Med 2009, 68(4):749-757.

21. King R, et al: Processes and outcomes of HIV serostatus disclosure to sexual partners among people living with HIV in Uganda. AIDS Behav 2008, 12(2):232-243.

22. Moore AR, Oppong J: Sexual risk behavior among people living with HIV/ AIDS in Togo. Social Sci Med 2007, 64(5):1057-1066.

23. Kanniappan S, Jeyapaul MJ, Kalyanwala S: Desire for motherhood: exploring HIV-positive women's desires, intentions and decision-making in attaining motherhood. AIDS Care 2008, 20(6):625-630.

24. NACC, UNGASS on HIV and AIDS Country Report: Kenya National AIDS Control Council: Nairobi; 2010.

25. Watters J, Biernacki P: Targeted sampling: Options for the study of hidden populations. Soc Probl 1989, 36(4):416-430.
26. Rothenberg R: Commentary: Sampling in Social Networks. Connections 1995, 18(1):104-110.

27. Magnani R, et al: Review of sampling hard-to-reach and hidden population for HIV surveillance. AIDS 2005, 19(Supplement 2):S67-S72.

28. Kaai $S$, et al: Perceived stigma among patients receiving antiretroviral treatment: a prospective randomised trial comparing an m-DOT strategy with standard-of-care in Kenya. SAHARA J 2010, 7(2):62-70.

29. Sarna $A$, et al: Short- and long-term efficacy of modified directly observed antiretroviral treatment in Mombasa, Kenya: a randomized trial. J Acquir Immune Defic Syndr 2008, 48(5):611-619.

30. Berger BE, Ferrans CE, Lashley FR: Measuring stigma in people with HIV: psychometric assessment of the HIV stigma scale. Res Nurs Health 2001, 24(6):518-529.

31. Kline A, Van Landingham M: HIV-infected women and sexual risk reduction: the relevance of existing models of behavior change. AIDS Educ Prev 1994, 6(5):390-402.

32. VandeVen P, et al: A scale of optimism-scepticism in the context of HIV treatments. AIDS Care 2000, 12(2):171-176

33. Greenland S, Rothman KJ: Precision and Statistics in Epidemiologic Studies [Chapter 10:]. Modern Epidemiology. 3 edition. Lipincott Williams and Wilkins. 758; 2008.

34. Pettifor A, et al: Continued High Risk Sexual Behavior Following Diagnosis with Acute HIV Infection in South Africa and Malawi: Implications for Prevention. AIDS Behav 2010

35. Loubiere $\mathrm{S}$, et al: HIV disclosure and unsafe sex among HIV-infected women in Cameroon: results from the ANRS-EVAL study. Socical Sci Med 2009, 69(6):885-891.

36. Eaton $L$, et al: HIV transmission risk among HIV seroconcordant and serodiscordant couples: dyadic processes of partner selection. AIDS Behav 2009, 13(2):185-195.

37. Wagner $\mathrm{G}$, et al: Factors associated with condom use among HIV clients in stable relationships with partners at varying risk for HIV in Uganda. AIDS Behav 2010, 14(5):1055-1065.

38. Bursac Z, et al: A Purposeful Selection of Macro Variables for Logistic Regression. SAS Global Forum 2007.

39. Marcellin $F$, et al: Higher risk of unsafe sex and impaired quality of life among patients not receiving antiretroviral therapy in Cameroon: results from the EVAL survey (ANRS 12-116). AIDS 2010, 24(suppl 1):S17-S25.

40. Randolph ME, et al: Sexual pleasure and condom use. Arch Sex Behav 2007, 36(6):844-848.

41. Conley T, Collins B: Difference between condom users and condom nonusers in their multidimensional condom attitudes. $J$ Appl Soc Psychol 2005, 35:603-620.

42. Rutenberg N, Baek C: Field experiences integrating family planning into programs to prevent mother-to-child transmission of HIV. Stud Fam Plann 2005, 36(3):235-245.

43. Church K, Mayhew SH: Integration of STI and HIV prevention, care, and treatment into family planning services: a review of the literature. Stud Fam Plan 2009, 40(3):171-186.

44. Kalichman S, Simbayi L: Sexual exposure to blood and increased risks for heterosexual HIV transmission in Cape Town, South Africa. Afr J Reprod Health 2004, 8(2):55-58

45. Tanfer $K$, Aral $\mathrm{S}$ : Sexual intercourse during menstruation and selfreported sexually transmitted disease history among women. Sex Transm Dis 1996, 23(5):395-401.

46. Geibel $\mathrm{S}$, et al: Factors associated with self-reported unprotected anal sex among male sex workers in Mombasa, Kenya. Sex Transm Dis 2008, 35(8):746-752.

47. Geibel $S$, et al: Are you on the market?: a capture-recapture enumeration of men who sell sex to men in and around Mombasa, Kenya. AIDS 2007, 21(10):1361-1362.

48. Kalichman $\mathrm{S}$, et al: Heterosexual anal intercourse among community and clinical settings in Cape Town, South Africa. Sex Transm Infect 2009, 85(6):411-415.

49. Kalichman S, Simbayi L, Cain D: HIV transmission risk behaviors among HIV seropositive sexually transmitted infection clinic patients in Cape Town, South Africa. Eur J Public Health 2010, 20(2):202-206.

50. Obermeyer C: Reframing research on sexual behavior and HIV. Stud Fam Plann 2005, 36(1):1-12. 
51. Ellish NJ, et al: Reliability of partner reports of sexual history in a hetersexual population at a sexually transmitted diseases clinic. Sex Transm Dis 1996, 23(6):446-452.

52. Minnis A, et al: Biomarker validation of reports of recent sexual activity: results of a randomized controlled study in Zimbabwe. Am J Epidemiol 2009, 170(7):918-924.

doi:10.1186/1742-6405-9-9

Cite this article as: Sarna et al:: Sexual behavior of HIV-positive adults not accessing HIV treatment in Mombasa, Kenya: Defining their prevention needs. AIDS Research and Therapy 2012 9:9.

Submit your next manuscript to BioMed Central and take full advantage of:

- Convenient online submission

- Thorough peer review

- No space constraints or color figure charges

- Immediate publication on acceptance

- Inclusion in PubMed, CAS, Scopus and Google Scholar

- Research which is freely available for redistribution

Submit your manuscript at www.biomedcentral.com/submit
() Biomed Central 